PROCEEDINGS OF THE

AMERICAN MATHEMATICAL SOCIETY

Volume 135, Number 7, July 2007, Pages 2051-2054

S 0002-9939(07)08660-1

Article electronically published on March 2, 2007

\title{
A SHARP INEQUALITY FOR THE LOGARITHMIC COEFFICIENTS OF UNIVALENT FUNCTIONS
}

\author{
OLIVER ROTH
}

(Communicated by Juha M. Heinonen)

Dedicated to the memory of Professor Nikolaos Danikas

ABStRaCt. We prove the sharp inequality

$$
\sum_{k=1}^{\infty}\left(\frac{k}{k+1}\right)^{2}\left|c_{k}(f)\right|^{2} \leq 4 \sum_{k=1}^{\infty}\left(\frac{k}{k+1}\right)^{2} \frac{1}{k^{2}}=\frac{2 \pi^{2}-12}{3}
$$

for the logarithmic coefficients $c_{k}(f)$ of a normalized univalent function $f$ in the unit disk.

\section{INTRODUCTION}

Let $\mathcal{S}$ denote the set of univalent functions $f$ in the unit disk $\mathbb{D}:=\{z \in \mathbb{C}$ : $|z|<1\}$ normalized by $f(0)=0$ and $f^{\prime}(0)=1$. The logarithmic coefficients $c_{k}(f)$ of a function $f \in \mathcal{S}$ are defined 11 by

$$
\log \frac{f(z)}{z}=\sum_{k=1}^{\infty} c_{k}(f) z^{k} .
$$

The celebrated de Branges' inequalities (the former Milin conjecture) for univalent functions state that

$$
\sum_{k=1}^{n}(n-k+1) k\left|c_{k}(f)\right|^{2} \leq 4 \sum_{k=1}^{n} \frac{n+1-k}{k}, \quad n=1,2,3, \ldots,
$$

with equality if and only if

$$
f(z)=\frac{z}{\left(1-e^{i \theta} z\right)^{2}}, \quad \theta \in \mathbb{R} ;
$$

see, for instance, [5, 6]. In addition to providing a proof of the Bieberbach conjecture the de Branges' inequalities are also the source of many other interesting inequalities involving logarithmic coefficients of univalent functions such as

$$
\sum_{k=1}^{\infty}\left|c_{k}(f)\right|^{2} \leq 4 \sum_{k=1}^{\infty} \frac{1}{k^{2}}=\frac{2 \pi^{2}}{3}
$$

Received by the editors September 13, 2005 and, in revised form, January 31, 2006.

2000 Mathematics Subject Classification. Primary 30C50; Secondary 30A10.

Key words and phrases. Univalent functions, logarithmic coefficients, de Branges' weight functions.

${ }^{1}$ We use the notation employed in [6], which differs from the notation in [3, 1, 4].

(C)2007 American Mathematical Society 
cf. [1, 4. Note that (1.2) follows quite easily from (1.1) using summation by parts. In this note we employ a more refined technique that permits us to prove sharp inequalites for logarithmic coefficients of univalent functions which do not directly follow from the de Branges' inequalities (1.1) and summation by parts. This method is based on summation by parts applied to (1.1) combined with a study of de Branges' differential system; see (2.4) below. As an illustration of this technique we establish the following best possible estimate.

Theorem 1.1. Let $f \in \mathcal{S}$. Then

$$
\sum_{k=1}^{\infty}\left(\frac{k}{k+1}\right)^{2}\left|c_{k}(f)\right|^{2} \leq 4 \sum_{k=1}^{\infty}\left(\frac{k}{k+1}\right)^{2} \frac{1}{k^{2}}
$$

Equality holds in (1.3) for $f \in \mathcal{S}$ if and only if

$$
f(z)=\frac{z}{\left(1-e^{i \theta} z\right)^{2}}, \quad \theta \in \mathbb{R} .
$$

Inequality (1.3) yields the sharp form of an inequality due to Danikas and Ruscheweyh [2, Corollary 4.2]. They introduced the operator

$$
\mathcal{T}_{f}(z):=\int_{0}^{z} \frac{\xi f^{\prime}(\xi)}{f(\xi)} d \xi=z+\sum_{k=1}^{\infty} \frac{k}{k+1} c_{k}(f) z^{k+1}, \quad f \in \mathcal{S},
$$

and conjectured that $\mathcal{T}_{f} \in \mathcal{S}$ for every $f \in \mathcal{S} \mathbb{Q}$ Theorem 1.1 implies the sharp inequality

$$
\left\|\mathcal{T}_{f}\right\|_{2}^{2}:=\lim _{r \rightarrow 1} \frac{1}{2 \pi} \int_{0}^{2 \pi}\left|\mathcal{T}_{f}\left(r e^{i t}\right)\right|^{2} d t \leq \frac{2 \pi^{2}-12}{3}+1 \approx 3.57974
$$

for the square of the $H^{2}$-norm of $\mathcal{T}_{f}$ improving the bound $1+2 \pi^{2} / 3 \approx 7.57974$ given in [2], which is based on (1.2).

\section{ProOF}

We first prove inequality (1.3). Fix $f \in \mathcal{S}$. Using summation by parts a lengthy but straightforward computation shows

$$
\sum_{k=1}^{\infty}\left(\frac{k}{k+1}\right)^{2}\left|c_{k}(f)\right|^{2}=\sum_{n=1}^{\infty} \lambda_{n}\left(\sum_{k=1}^{n}(n-k+1) k\left|c_{k}(f)\right|^{2}\right),
$$

where

$$
\lambda_{n}=\frac{n}{(n+1)^{2}}-2 \frac{n+1}{(n+2)^{2}}+\frac{n+2}{(n+3)^{2}}, \quad n=1,2, \ldots
$$

Thus we need to prove

$$
\sum_{n=1}^{\infty} \lambda_{n}\left(\sum_{k=1}^{n}(n-k+1) k\left|c_{k}(f)\right|^{2}\right) \leq 4 \sum_{n=1}^{\infty} \lambda_{n}\left(\sum_{k=1}^{n} \frac{n+1-k}{k}\right) .
$$

\footnotetext{
${ }^{2}$ This conjecture is still open.
} 
Note that $\lambda_{1}=-1 / 144<0$, but $\lambda_{n}>0$ for every $n \geq 2$. Therefore, in view of de Branges' inequalities (1.1), it suffices to prove that

$$
\sum_{n=1}^{N} \lambda_{n}\left(\sum_{k=1}^{n}(n-k+1) k\left|c_{k}(f)\right|^{2}\right) \leq 4 \sum_{n=1}^{N} \lambda_{n}\left(\sum_{k=1}^{n} \frac{n+1-k}{k}\right)
$$

for some integer $N \geq 1$. Now (2.2) is equivalent to

$$
\sum_{k=1}^{N}\left(k\left|c_{k}(f)\right|^{2}-\frac{4}{k}\right) \mu_{k} \leq 0
$$

where

$$
\mu_{k}:=\sum_{j=1}^{N-k+1} j \lambda_{j+k-1} .
$$

Thus it remains to establish (2.3) for some integer $N \geq 1$.

For this we consider the de Branges' system of ordinary differential equations

$$
\begin{aligned}
\tau_{k}(t)-\tau_{k+1}(t) & =-\frac{\tau_{k}^{\prime}(t)}{k}-\frac{\tau_{k+1}^{\prime}(t)}{k+1}, \quad k=1, \ldots, N, \\
\tau_{k}(0) & =\mu_{k},
\end{aligned}
$$

where $\tau_{N+1}(t) \equiv 0$, and define the function

$$
\varphi(t):=\sum_{k=1}^{N}\left(k\left|c_{k}(t)\right|^{2}-\frac{4}{k}\right) \tau_{k}(t)
$$

Here the $c_{k}(t)$ 's are the logarithmic coefficients of $e^{-t} f(z, t) \in \mathcal{S}$, and $f(z, t)$ is a normalized Löwner chain with $f(z, 0)=f(z)$. Then (see [],,$(3.11)$ )

$$
\varphi^{\prime}(t)=-\sum_{k=1}^{N}\left|b_{k+1}(t)-b_{k}(t)+2\right|^{2} \frac{\tau_{k}^{\prime}(t)}{k}
$$

and $\varphi(t) \rightarrow 0$ as $t \rightarrow \infty$. Here, the $b_{k}$ 's are certain functions depending on the particular choice of the Löwner chain $f(z, t)$. Consequently, if we can show that for some integer $N \geq 1$ the solutions to (2.4) are monotononically decreasing on the interval $[0, \infty)$, then the limit relation

$$
\lim _{t \rightarrow \infty} \varphi(t)=0
$$

implies $\varphi(0) \leq 0$, which is exactly what we want to prove.

Now, the solutions of the differential system (2.4) for $N=1,2,3, \ldots$ can be found by direct calculation. It turns out that $\tau_{1}^{\prime}(0)>0$ for $N=1,2,3,4$, so the weight function $\tau_{1}$ is not monotonically decreasing on the interval $[0, \infty)$ in these 
cases. However, for $N=5$ one finds

$$
\begin{aligned}
& \tau_{1}(t)=\frac{99750-378644 e^{t}+581880 e^{2 t}-468720 e^{3 t}+209609 e^{4 t}}{705600 e^{5 t}}, \\
& \tau_{2}(t)=\frac{-28500+94661 e^{t}-116376 e^{2 t}+58590 e^{3 t}}{176400 e^{5 t}}, \\
& \tau_{3}(t)=\frac{21375-54092 e^{t}+38792 e^{2 t}}{235200 e^{5 t}}, \\
& \tau_{4}(t)=\frac{-9500+13523 e^{t}}{352800 e^{5 t}}, \\
& \tau_{5}(t)=\frac{95}{28224 e^{5 t}},
\end{aligned}
$$

and it is easy to verify that the weights $\tau_{1}, \ldots, \tau_{5}$ are monotonically decreasing on the entire interval $[0, \infty)$. This proves (2.3) for $N=5$ and, as we have seen above, completes the proof of inequality (1.3).

It remains to establish the equality statement of Theorem 1.1. If $f \in \mathcal{S}$ is of the form (1.4), then $\left|c_{k}(f)\right|=2 / k$ for every $k \geq 1$, so equality holds in (1.3). On the other hand, if $f \in \mathcal{S}$ is not of the form (1.4), then the same argument as in 6 , Paragraph 4] shows that

$$
\sum_{k=1}^{5}\left(k\left|c_{k}(f)\right|^{2}-\frac{4}{k}\right) \mu_{k}<0
$$

where

$$
\mu_{k}:=\sum_{j=1}^{5-k+1} j \lambda_{j+k-1}, \quad k=1, \ldots, 5 .
$$

Thus strict inequality holds in (2.2) for $N=5$, and so, in view of (1.1), also in (2.1) and in (1.3).

\section{REFERENCES}

[1] Andreev, V. V., Duren, P. L., Inequalities for logarithmic coefficients of univalent functions and their derivatives, Indiana Univ. Math. J. 37, No. 4, 721-733, 1988. MR0982827 (90c:30026)

[2] Danikas, N., Ruscheweyh, St., Semi-convex hulls of analytic functions in the unit disk, Analysis, No. 4, 309-318, 1999. MR1743524 (2001c:30020)

[3] Duren, P. L., Univalent functions, Springer (1983). MR0708494 (85j:30034)

[4] Duren, P. L., Leung, Y. L., Logarithmic coefficients of univalent functions, J. Anal. Math. 36, 36-43, 1979. MR0581799(81i:30018)

[5] de Branges, L., A proof of the Bieberbach conjecture, Acta Math. 154, 137-152, 1985. MR0772434 (86h:30026)

[6] FitzGerald, C. H., Pommerenke, Chr., The de Branges theorem on univalent functions, Trans. Amer. Math. Soc. 290 No. 2, 683-690, 1985. MR0792819 (87b:30023)

Mathematisches Institut, Universität WÜrzburg, D-97074 Würzburg, Germany

E-mail address: roth@mathematik.uni-wuerzburg.de 